\title{
CP-DSSS: An OFDM Compatible Variable Rate Modulation for 5G and Beyond
}

\section{October 2020}

Behrouz Farhang-Boroujeny, Arslan J Majid, Hussein Moradi 


\section{DISCLAIMER}

This information was prepared as an account of work sponsored by an agency of the U.S. Government. Neither the U.S. Government nor any agency thereof, nor any of their employees, makes any warranty, expressed or implied, or assumes any legal liability or responsibility for the accuracy, completeness, or usefulness, of any information, apparatus, product, or process disclosed, or represents that its use would not infringe privately owned rights. References herein to any specific commercial product, process, or service by trade name, trade mark, manufacturer, or otherwise, does not necessarily constitute or imply its endorsement, recommendation, or favoring by the U.S. Government or any agency thereof. The views and opinions of authors expressed herein do not necessarily state or reflect those of the U.S. Government or any agency thereof. 


\section{CP-DSSS: An OFDM Compatible Variable Rate Modulation for $\mathbf{5 G}$ and Beyond}

Behrouz Farhang-Boroujeny, Arslan J Majid, Hussein Moradi

October 2020

Idaho National Laboratory
Idaho Falls, Idaho 83415

http://www.inl.gov

Prepared for the U.S. Department of Energy

Under DOE Idaho Operations Office

Contract LDRD 19A39-137 


\title{
CP-DSSS: An OFDM Compatible Variable Rate Modulation for $5 \mathrm{G}$ and Beyond
}

\author{
Behrouz Farhang-Boroujeny $^{\dagger}$, Arslan Majid ${ }^{\ddagger}$, and Hussein Moradi ${ }^{\ddagger}$ \\ ${ }^{\dagger}$ ECE Department, University of Utah, USA ${ }^{\ddagger}$ Idaho National Laboratory, Idaho Falls, USA
}

\begin{abstract}
Cyclic prefix direct sequence spread spectrum (CPDSSS) is a recently proposed waveform that has been designed for coexistence with OFDM (orthogonal frequency division multiplexing) in the same or a pair of parallel networks. The first contribution of this paper is to present equations that reveal similarities and differences of CP-DSSS and OFDM. Furthermore, we show that the channel model of CP-DSSS reduces to that of a cyclic prefixed block-wise single carrier modulation (CP-SCM). However, unlike CP-SCM whose symbol rate is always equal to the transmission bandwidth, CP-DSSS can be adopted to any symbol rate equal to or smaller than the transmission bandwidth. This reduction in symbol rate allows CP-DSSS power spectral density to drop to an arbitrarily low level, hence, facilitates it coexistence as a secondary waveform in a network of primary users. The peak-to-average power ratio (PAPR) of CP-DSSS is also explored and a method for reducing it is proposed.
\end{abstract}

\section{INTRODUCTION}

Cyclic prefix direct sequence spread spectrum (CP-DSSS) is a novel spread spectrum waveform that has been recently proposed [1]-[6] and has the potential of solving some of the key objectives of ultra-reliable low latency communications (URLLC) and massive machine type communications (mMTC) that have been noted in 5G NR but reliable solutions to them still to be explored/found.

In [1] and [2], CP-DSSS was introduced as a secondary signaling method for sending a small number of control bits within each OFDM symbol interval concurrent with other communication signals within the network to enable resource allocation for URLLC communications. In [3], the feasibility of this signaling method in a $3 \mathrm{GPP}$ proposed industrial setting was explored and its reliable performance was further confirmed.

In [4]-[6], the CP-DSSS waveform was looked at as a data channel. The emphasis of these works is to use CP-DSSS as a secondary waveform that may co-exist with wireless activities in a primary network (e.g., in an LTE or 5G NR network). The general finding in these works is that CP-DSSS, being a spread spectrum technique, can be tuned to operate reliably at some low signal-to-interference-plus-noise ratio (SINR) and, accordingly, some low data rates which may be acceptable to

This manuscript has been authored by Battelle Energy Alliance, LLC under Contract No. DE-AC07-05ID14517 with the U.S. Department of Energy. The United States Government retains and the publisher, by accepting the paper for publication, acknowledges that the United States Government retains a nonexclusive, paid-up, irrevocable, world-wide license to publish or reproduce the published form of this manuscript, or allow others to do so, for United States Government purposes. STI Number: INL/CON-20-58521. the needs of machine-type communications. It is further argued in [4]-[6] that by forming femtocells within a cellular network and employing multiple antennas at each femtocell gateway (FGW)/base station, the secondary network interference to primary users/network may be kept at a minimum level. Here, we wish to further note that CP-DSSS may be considered as an independent waveform for transmission of both control and data signals in variety of environments beyond what have been studied in the above referenced works.

The goal of this paper is to dig into more details of CPDSSS and explore its similarities and differences with the class of block-wise single carrier modulation techniques. This class of modulation techniques divide a single carrier stream into blocks to allow efficient processing in the frequency domain using the fast Fourier transform (FFT) and the inverse FFT (IFFT). The celebrated methods of overlap and save and the addition of a cyclic prefix to each block (as in orthogonal frequency division multiplexing (OFDM)) are commonly adopted, e.g., [7]-[10]. The latter, which may be called cyclic prefix single carrier modulation (CP-SCM) has great similarity with CP-DSSS. There is also a variation of the CP-SCM where the $\mathrm{CP}$ is replaced with a pseudo noise $(P N)$ extension. These variations are discussed in detail in [11].

Part of the study of this paper reveals that CP-DSSS has a channel model that is similar to that of CP-SCM. However, the spread spectrum nature of CP-DSSS allows its adoption as an underlay communication channel that may coexist with other communication signals within the same network. We also propose an effective method that may be used to control the peak-to-average power ratio (PAPR) of CP-DSSS to a level comparable to that of single carrier systems.

This paper is organized as follows. Section II presents the basic equation that reveals the similarities and differences that exist between OFDM, CP-SCM, and CP-DSSS. In particular, the equations that reveal the similarity of channel models of CP-SCM and CP-DSSS are presented. Moreover, the flexibility of CP-DSSS for reducing the data rate, hence, reducing the signal power, when it is used as a secondary waveform within a network of primary users is discussed. A method of reducing the PAPR of CP-DSSS, hence, making it an excellent choice for mMTC devices is presented in Section III. The concluding remarks of the paper are made in Section V.

\section{CP-DSSS VERSUS OFDM AND CP-SCM}

The CP-DSSS waveform follows the frame structure of OFDM. This is also the case for CP-SCM. Here, we present 
a summary of OFDM, CP-DSSS, and CP-SCM with the goal of revealing their similarities and differences among them.

\section{A. $O F D M$}

In OFDM, each signal frame carries a number of data symbols that modulate a set of complex-valued sinusoidal signals/tones. Furthermore, by adding a $\mathrm{CP}$ to each signal frame, the data carrying tones are made to correspond to a orthogonal basis set. Mathematically, a frame of synthesized OFDM signal, excluding the $\mathrm{CP}$, may be expressed as

$$
\mathbf{x}=\sum_{k} s_{k} \mathbf{f}_{k}
$$

where $k$ covers the set of active subcarriers and

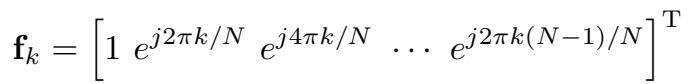

is a column vector of length $N$. In OFDM, the active tones are contiguous in $k$ and only a few subcarriers at the two sides of the band are suppressed to act as guard bands. Hence, the number of data symbols transmitted in each OFDM frame is smaller than $N$. One may note that the summation in (1) is, effectively, the well-known IFFT operation in OFDM and $N$ is the FFT/IFFT size. It may be further noted that the column vectors $\mathbf{f}_{k}$ correspond to an orthogonal basis set.

\section{B. $C P-D S S S$}

The construction (1) may be generalized by replacing the basis set $\mathbf{f}_{k}$ by an arbitrary orthogonal set $\boldsymbol{\eta}_{k}$. This leads to a synthesized signal vector

$$
\mathbf{x}=\sum_{k} s_{k} \boldsymbol{\eta}_{k} .
$$

In CP-DSSS $\boldsymbol{\eta}_{k}$ are a set of spreading gain vectors, of length $N$, which should be chosen to be orthogonal to each other. Hence, these gain vectors may be also thought as a basis set that carry the data symbols $s_{k}$. However, there is a fundamental difference between the basis set $\mathbf{f}_{k}$ of OFDM and the basis set $\boldsymbol{\eta}_{k}$ of CP-DSSS. While the OFDM basis set may be thought as a set of narrowband signals, the CP-DSSS basis set are chosen to be a set of broadband signals.

It turns out that the set of $\boldsymbol{\eta}_{k}$ that leads to an effective CPDSSS signal is obtained by selecting $\boldsymbol{\eta}_{0}=\mathbf{z}_{0}$, where $\mathbf{z}_{0}$ is a Zadoff-Chu (ZC) sequence of length $N$. We also define

$$
\mathbf{z}_{0}=\left[\begin{array}{c}
z_{0} \\
z_{1} \\
z_{2} \\
\vdots \\
z_{N-1}
\end{array}\right]
$$

and let $\mathbf{z}_{1}, \mathbf{z}_{2}, \cdots, \mathbf{z}_{N-1}$ be the circularly shifted versions of $\mathbf{z}_{0}$, defined as:

$$
\mathbf{z}_{1}=\left[\begin{array}{c}
z_{N-1} \\
z_{0} \\
z_{1} \\
\vdots \\
z_{N-2}
\end{array}\right] \mathbf{z}_{2}=\left[\begin{array}{c}
z_{N-2} \\
z_{N-1} \\
z_{0} \\
\vdots \\
z_{N-3}
\end{array}\right] \cdots \mathbf{z}_{N-1}=\left[\begin{array}{c}
z_{1} \\
z_{2} \\
z_{3} \\
\vdots \\
z_{0}
\end{array}\right]
$$

A key property of $\mathrm{ZC}$ sequences is that the set of vectors $\mathbf{z}_{0}, \mathbf{z}_{1}, \mathbf{z}_{2}, \cdots, \mathbf{z}_{N-1}$ are orthogonal to each other, i.e., for all values of $i$ and $j$ in the range of 0 to $N-1$ :

$$
\mathbf{z}_{i}^{\mathrm{H}} \mathbf{z}_{j}=0, \text { for and } i \neq j,
$$

where the superscript ' $\mathrm{H}$ ' denotes Hermitian (i.e., conjugate transpose). That is, this choice satisfies the orthogonality requirement of the basis set $\mathbf{z}_{k}$. Hence, $\boldsymbol{\eta}_{k}=\mathbf{z}_{k}$ is one possible choice to form a CP-DSSS waveform. This particular choice, as discussed below, leads to some special properties that makes CP-DSSS waveform a proper choice for many applications to $5 \mathrm{G}$ and beyond. Hence, for the rest of this document, we only consider the construction CP-DSSS signals according to the following equation

$$
\mathbf{x}=\sum_{k} s_{k} \mathbf{z}_{k} .
$$

In the sequel, we assume that $\mathbf{z}_{0}$ is normalized to the length of unity. With this additional property, the orthogonality property of $\mathrm{ZC}$ sequences that was just mentioned may be summarized as

$$
\mathbf{z}_{i}^{\mathrm{H}} \mathbf{z}_{j}= \begin{cases}1, & i=j \\ 0, & i \neq j .\end{cases}
$$

We further note that the summation over $k$ in (6) may cover a subset of indices $\{0,1, \cdots, N-1\}$. In other words, the number of symbols $s_{k}$ transmitted over each frame of CPDSSS waveform can be smaller than $N$. This allows keeping the symbol rate smaller than the transmission bandwidth, which is typical of all spread spectrum systems. An extreme example of this is the control signaling method that has been discussed in [2] and [3], where only one or two bits are transmitted over each CP-DSSS frame. In [4]-[6] and here, we consider the case where $N$ is divisible by an integer $L$, and $N / L$ symbols are transmitted over each CP-DSSS frame. Calling these symbols $s_{0}, s_{1}, \cdots, s_{N / L-1}$, the synthesized vector $\mathrm{x}$ of (6) may be expressed as

$$
\mathbf{x}=\mathbf{Z E s}
$$

where $\mathbf{s}$ is a column vector of length $N / L$ with elements of $s_{0}, s_{1}, \cdots, s_{N / L-1}, \mathbf{E}$ is an expander matrix that adds $L-1$ zeros after each element of $\mathbf{s}$ to make a column vector of length $N$, and $\mathbf{Z}$ is an $N \times N$ matrix with columns of $\mathbf{z}_{0}, \mathbf{z}_{1}, \mathbf{z}_{2}, \cdots, \mathbf{z}_{N-1}$

After adding a $\mathrm{CP}$ to $\mathbf{x}$ and passing it through a channel with the impulse response $\mathbf{h}$, the received signal, after removing the $\mathrm{CP}$, will find the following form

$$
\mathbf{y}=\mathbf{H Z E s}+\mathbf{v}
$$

where $\mathbf{v}$ is the channel noise vector and $\mathbf{H}$ is a circulant matrix of size $N \times N$ whose first column is the channel impulse response $\mathbf{h}$ appended with zeros to be extend to the length $N$. Here, to allow the formulation (8), we have assumed that the length of $\mathbf{h}$, denoted by $L_{h}$, is smaller than or equal to the $\mathrm{CP}$ length. 


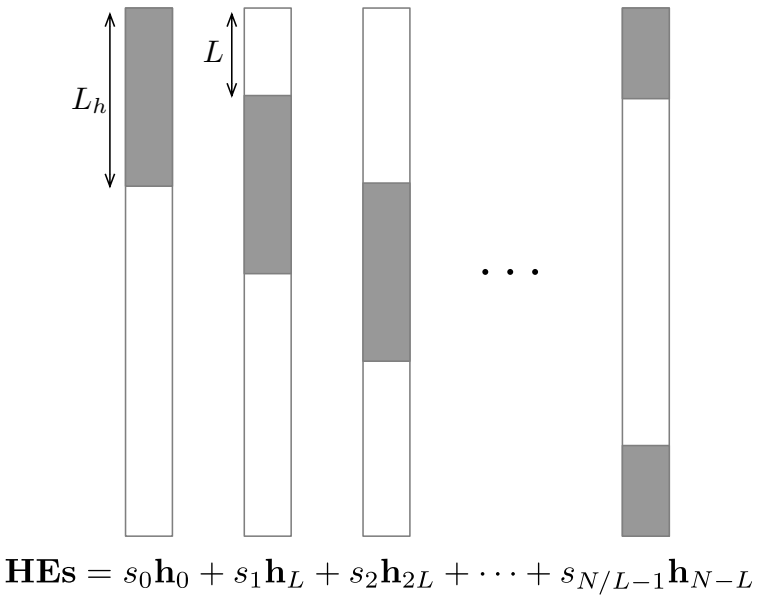

Fig. 1. A pictorial presentation of HEs. The vertical boxes show the relevant columns of $\mathbf{H}$, with the gray parts indicating the non-zero terms.

Dispreading the received signal vector $\mathbf{y}$, we obtain

$$
\begin{aligned}
\tilde{\mathbf{y}} & =\mathbf{Z}^{\mathrm{H}} \mathbf{y} \\
& =\mathbf{Z}^{\mathrm{H}} \mathbf{H Z E s}+\tilde{\mathbf{v}} .
\end{aligned}
$$

Noting that the matrices $\mathbf{Z}^{\mathrm{H}}, \mathbf{H}$, and $\mathbf{Z}$ are circulant, and circulant matrices are commutable in multiplication, and recalling that $\mathbf{Z}^{\mathrm{H}} \mathbf{Z}=\mathbf{I}$, one will find that (10) reduces to

$$
\tilde{\mathbf{y}}=\mathbf{H E s}+\tilde{\mathbf{v}}
$$

Fig. 1 visualizes the first term on the right-hand side of (11), i.e., HEs. As seen, the inter-symbol symbol interference (ISI) may occur, if $L_{h}>L$. On the other hand, when $L_{h} \leq L$, there will be no ISI.

\section{C. $C P-S C M$}

In CP-SCM, the transmit signal vector $\mathbf{x}$ is simply a symbol vector $\mathbf{s}$ of length $N$, i.e.,

$$
\begin{aligned}
\mathbf{x} & =\mathbf{s} \\
& =\sum_{k} s_{k} \mathbf{i}_{k}
\end{aligned}
$$

where $\mathbf{i}_{k}$ are the set of standard basis vectors of an $N$ dimensional Euclidean space, i.e., $\mathbf{i}_{k}$ is a column vector with zero entries at all positions, except an entry of one at position $k$. Construction (12) has the same form as the general construction (2), and construction (6) of CP-DSSS. The difference here is that CP-SCM uses the trivial standard basis vectors $\mathbf{i}_{0}, \mathbf{i}_{1}, \cdots, \mathbf{i}_{N-1}$.

As in the case of CP-DSSS, here, also, a CP is added to $\mathbf{x}$ before transmission. At the receiver, after removing the $\mathrm{CP}$, one obtains the received signal vector

$$
\mathbf{y}=\mathbf{H s}+\mathbf{v}
$$

where $\mathbf{H}$ is the circulant channel matrix that was defined above and $\mathbf{v}$ is the channel noise vector.

\section{Comparisons}

General observation made above was that a common feature of OFDM, CP-DSSS, and CP-SCM is that they all transmit a number data symbols, jointly, by modulating a set of orthogonal basis vectors. The difference between them come from the variation of basis vectors that they use. OFDM basis vectors are a set of single tones. This, in turns, leads to concentration of each symbol energy over narrow band. $\mathrm{CP}$ DSSS and CP-SCM, on the other hand, use basis vectors that are broadband and, hence, spread the energy of each symbol over the full-band of transmission. Furthermore, while in CPDSSS the signal energy of each data symbols is spread across the respective data frame, in CP-SCM each data symbol is localized in time. To emphasize more: (i) OFMD localizes each data symbol at a tone (equivalently, at a frequency point), but spread it in time across the respective data fram; (ii) SCSCM localizes each data symbol at sample point in time, but spread it in frequency across the full-band of transmission; and (iii) CP-DSSS spreads each data symbol across both time and frequency.

To further reveal the differences between CP-DSSS and CPSCM and the advantages that they may offer each other, we note that (11) reduces to (13), if the expander parameter $L$ is set equal one. This is because when $L=1, \mathbf{E}$ will be the identity matrix. Moreover, one may note that in CP-SCM, also, one may choose to transmit a symbol vector s of length $N / L$, after expanding it to the length $N$. In that case, the transmit signal vector will be

$$
\mathbf{x}=\mathbf{E s}
$$

and at the receiver, we will have

$$
\mathbf{y}=\mathbf{H E s}+\mathbf{v} \text {. }
$$

This has the same form as (11). We may further note here that the construction $\mathbf{x}$, in the current literature, is limited to (12). Here, we have introduced (14) to emphasis additional flexibilities and advantages that are offered by CP-DSSS.

Next, we compare the signal vector (14) of CP-SCM and the signal vector (8) of CP-DSSS. As $L$ increases, one may find that the signal vector of CP-SCM become sparse with all of its energy concentrated in $1 / L$ fraction of its samples. This clearly leads to a signal with an undesirable high peakto-average power ratio (PAPR). The signal vector CP-DSSS, on the other hand, spreads the signal energy over all samples of the data frame, hence, may have the favorable property of a much lower PAPR. Nevertheless, one may note that when $L=1$, CP-SCM may offer a lower PAPR when compared with its CP-DSSS counterpart. We elaborate on these, in the next section, through some theoretical study and by presenting some numerical examples.

It may be further noted that the choice of $L>1$ may be of particular interest in multiuser use cases. Larger values of $L$ provide more processing gain for each user at a cost of some reduction in data rate. In a multiuser scenario, this allows more users to communicate simultaneously, hence, lead to some fairness to all the users in the network and, furthermore, may 
lead to higher sum rate for the whole network. Theoretical studies and numerical examples that quantify this feature of CP-DSSS is presented in [6]. We may further note that a higher processing gain translates a lower transmit power that, in general, is of interest, particularly for mMTC applications.

\section{PAPR}

We first recall that what makes the PAPR of OFDM large is directly related to signal construction (1). Here, the signal vector $\mathbf{x}$ is constructed by adding some random phases and amplitudes to the set of orthogonal vectors $\mathbf{f}_{k}$ and adding the results. This, for a large $N$, converges to a Gaussian like process with some large amplitude samples which, in turn, translates to a large PAPR. Clearly, the same is true for the CP-DSSS signal construction (6). One may thus conclude that CP-DSSS faces the same PAPR problem as OFDM. However, there is a point to be noted and we wish to emphasize here. Clipping a signal (being, OFDM or CP-DSSS) to control its PAPR to a reasonable level will introduce a small amount of noise across the spectrum whose presence will be destructive only when the operating SNR is relatively high. At lower SNR regime, one may find that signal clipping has very little impact on the performance of a CP-DSSS/OFDM-based communication link and, contrary to what one may think, in some cases may lead to a performance improvement. Here, we quantify this performance degradation/improvement through some theoretical explanation and presentation of some numerical results. We further note that CP-DSSS operation in low SNR regime is of particular interest in many use cases of this waveform as discussed in [5] and [6]. Considering this point, the PAPR reduction method that is introduced below will be presented with emphasis on CP-DSSS waveform, even though it is equally extendable to OFDM as well.

To structure our study and discuss how one may synthesize a CP-DSSS signal for controlling the PAPR, we consider the transmitter system block diagram shown in Fig. 2. Here, first, the data symbol vector $\mathbf{s}$ is passed to the CP-DSSS construction block that produces the signal vector $\mathbf{x}$ according to (8). The result is then passed through a clipping block that clips the elements of $\mathrm{x}$ according to the following equation:

$$
\bar{x}_{k}= \begin{cases}\eta \sigma_{x} e^{j \angle\left(x_{k}\right)}, & \text { if }\left|x_{k}\right|>\eta \sigma_{x} \\ x_{k}, & \text { otherwise }\end{cases}
$$

where $\sigma_{x}$ denotes the standard deviation of $x_{k}, \eta$ is a clipping parameter, and $\angle(x)$ denotes the angle of the complexvalued number $x$. The result is then $M$-fold upsampled and interpolated using an interpolation filter $h(t)$. A point to note here is that the choice of interpolation filter $h(t)$ can greatly impact the PAPR of the generated signal. This is discussed in some detail below. We further assume that this step delivers a continuous-time signal which is denoted by $x(t)$. Finally, $x(t)$ is cyclic prefixed and modulated to a radio frequency (RF) band to produce the continuous-time signal $x_{\mathrm{RF}}(t)$ for transmission.
The PAPR of CP-DSSS may be further reduced by replacing the clipping equation (16) with the constant-modulo (CM) signal

$$
\bar{x}_{k}=e^{j \angle\left(x_{k}\right)} \text {. }
$$

This preserves the phase of the elements of $\mathbf{x}$ and normalizes amplitude of them to unity. Clearly, both (16) and (17) introduce some distortion to the synthesized signal and thus perfect recovery of the transmitted symbols $s_{k}$ may not be possible, even in an ideal channel. The impact of the introduced distortion may be measured by the variance of introduced noise that will appear at the receiver output when it is passed through an ideal channel. This is evaluated below numerically. In the sequel, it is the PAPR of $x_{\mathrm{RF}}(t)$ that is of interest and, thus, will be presented.

There are different choices for the interpolation filter $h(t)$. It can be simply a lowpass filter of any arbitrary bandwidth. Such lowpass filter can combine the elements of the vector $\overline{\mathbf{x}}$, leading a Gaussian-like process which, in turn, undo the clipping effect that was desired. The use of a Nyquist filter that preserves the samples of $\overline{\mathbf{x}}$ at the interpolator output can be very effective. Moreover, numerical tests have revealed that the use of a square-root Nyquist filter is almost equally effective. Noting that the latter allows the use of a matched filter equivalent of it at the receiver, for maximizing noise suppression at the receiver, while allowing perfect recovery of transmitted symbols (in an ideal channel), the numerical results that we present here are for the case where a squareroot Nyquist filter is used for $h(t)$.

We recall that PAPR is often quantified by the complementary cumulative distribution function $(\mathrm{CCDF})$ of the signal of interest; here, $x_{\mathrm{RF}}(t)$. It presents the probability of $\left|x_{\mathrm{RF}}(t)\right|$ being greater than a threshold $\gamma$, as a function of $\gamma$. In addition, the relative size of $\gamma$ with respect to the standard deviation of $x_{\mathrm{RF}}(t)$, in decibel, is presented. Fig. 3 presents a set of such plots for CP-DSSS, for different choices of the clipping parameter $\eta$. Moreover, for comparison, the CCDF plots of the CP-SCM, for the choices of the expander parameter $L=1$ and 8 , are also presented. We further note that the CCDF plots of CP-DSSS are independent of $L$. The interpolation filter $h(t)$, as noted above, is a square-root Nyquist filter that is matched with the sample rate in the synthesized signal $\mathbf{x}$. The rolloff parameter of this filter is set to $\alpha=0.2$. The PAPR of both CP-DSSS and CP-SCM can be reduced by choosing a larger value of $\alpha$, but this has a direct impact on increasing the transmission bandwidth which, of course, is undesirable.

The general observation here, as was predicted above, is that CP-SCM with the expander parameter $L=1$ and CPDSSS clipped to a CM (according to (17)) have the lowest PAPR, with the latter performing slightly better. It is also observed that the PAPR of CP-SCM increases significantly as $L$ increases. CP-DSSS with no clipping, on the other hand, has a comparable PAPR to OFDM, but its PAPR may be reduced and brought to about 1 to $2 \mathrm{~dB}$ of that of CP-SCM by applying the clipping method that was introduced in (16). As also mentioned above, the clipping introduces some interference 


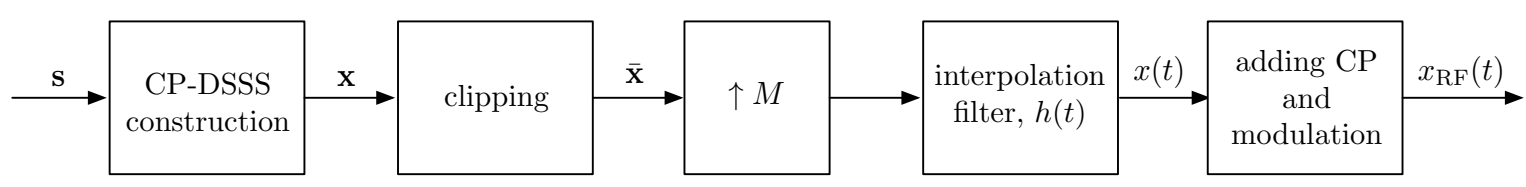

Fig. 2. Transmitter block diagram of CP-DSSS.

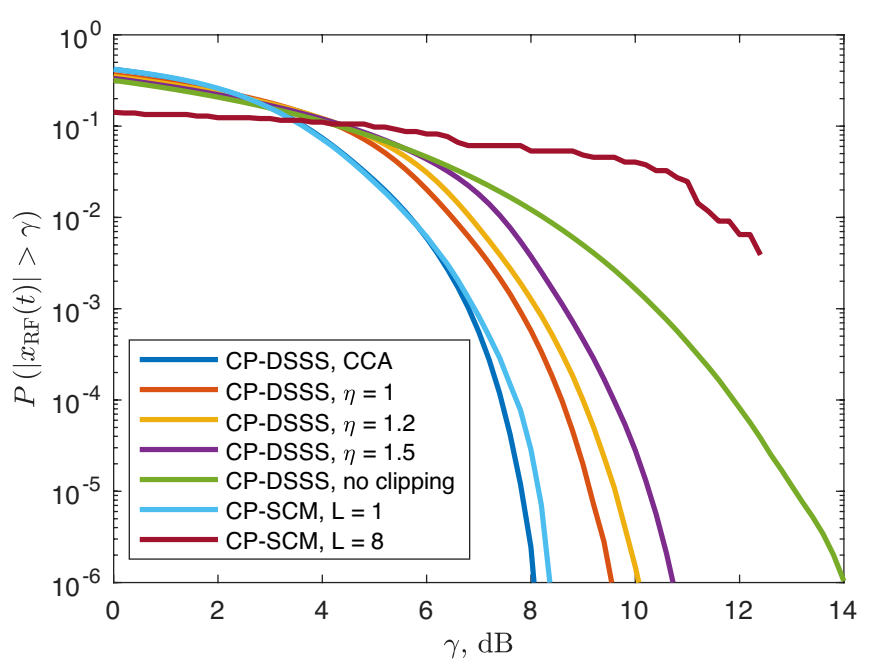

Fig. 3. CCDF plots for CP-DSSS with different clipping level, and CP-SCM for to choices of parameter $L$.

that, as long as it remains significantly lower than channel noise, may be tolerable. For the results presented here, the observed signal-to-interference ratio (SIR) at the output of the CP-DSSS detector were $6.5 \mathrm{~dB}$, when clipped to a CM, and $12 \mathrm{~dB}, 15 \mathrm{~dB}$, and $20 \mathrm{~dB}$ when (16) was applied and $\eta=1$, 1.2 , and 1.5 , respectively.

\section{CAPACITy Evaluation}

To get a better understanding of the clipping method that was presented above, here, we run some simulation to compare the capacity of CP-DSSS transceiver with and without clipping. The results are presented for the case where both transmitter and receiver are equipped with a single antenna. Similar observation is expected when multiple antennas are employed at base station and possibly multiple users are sharing the same spectrum, vis., in multiuser massive MIMO setup.

The results are presented for the case where $N=2048$ and $L=8$. The clipping parameter $\eta$ is set equal to one. The results are uplink and a matched filter detector is used at the base station. The results are based on 1000 independent random realization of a channel with exponential power delay profile with roll-off time constant 25 samples and a truncated length of 130 samples. As a measure of transmission capacity, the per symbol capacity of our setup is evaluated as

$$
C=\log _{2}(1+\rho) \text { bits per symbol }
$$

where $\rho$ is signal-to-interference plus noise ratio (SINR). The transmitted symbols, $s$, are chosen from a quadrature amplitude modulation (QAM) constellation and the SINR at the receiver output is calculated as

$$
\rho=\frac{\mathbb{E}\left[|s|^{2}\right]}{\mathbb{E}\left[|\hat{s}-s|^{2}\right]}
$$

where $\mathbb{E}[\cdot]$ denotes statistical expectation and $\hat{s}$ is the estimate of $s$ at the receiver output.

The simulation results are presented in Fig. 4. As one would expect, at higher values of SNR, clipping incurs some loss in performance. On the other hand, at lower SNR, it results in some performance gain. This latter observation, which may be counter intuitive, can be explained as follows. Removing high peaks of a synthesized CP-DSSS signal, through clipping, brings down the signal power to a lower value. This reduces the SNR, for a given level of channel noise. At lower values of SNR, when clipping noise is negligible compared to the channel noise, the reduced SNR shifts the capacity curve to the left side, as observed in Fig. 4. At higher values of SNR, on the other hand, the clipping noise effect on SINR is more significant and, thus leads to some reduction of the capacity. One may further note that at high SNR, the channel noise vanishes to a negligible level and, thus, the SINR at the receiver output is determined by inter-symbol interference (ISI) and and clipping noise. Here, given the channel length of 130 , the ISI will dominate and thus the degradation due to clipping noise is relatively small; about $20 \%$ reduction in capacity is observed. What is more important to note is that the greatest benefit of the spread spectrum nature of CP-DSSS is in cases that it is used as an underlay channel for operation of femtocells that serve a high density of mMTC devices. This is discussed in great detail in [4]-[6].

\section{CONCLUSION}

This paper contrasted CP-DSSS waveform with OFDM and CP-SCM and showed their similarities and differences. It was noted that while in OFDM each data symbol modulates a tone, hence, transmitted over a narrow band, in CP-DSSS all data symbols are spread over the full band of transmission. When compared to CP-SCM, it was found that the channel model of CP-DSSS is similar to that of CP-SCM, however, while CPDSSS spreads the signal energy of each data symbol across the respective data frame, in $\mathrm{CP}-\mathrm{SCM}$ signal energy of each data symbol is concentrated at a particular point in time. It was shown that this gives a great PAPR advantage to CP-DSSS in certain use cases of mMTC where a small number of data symbols may be transmitted over each data frame. 


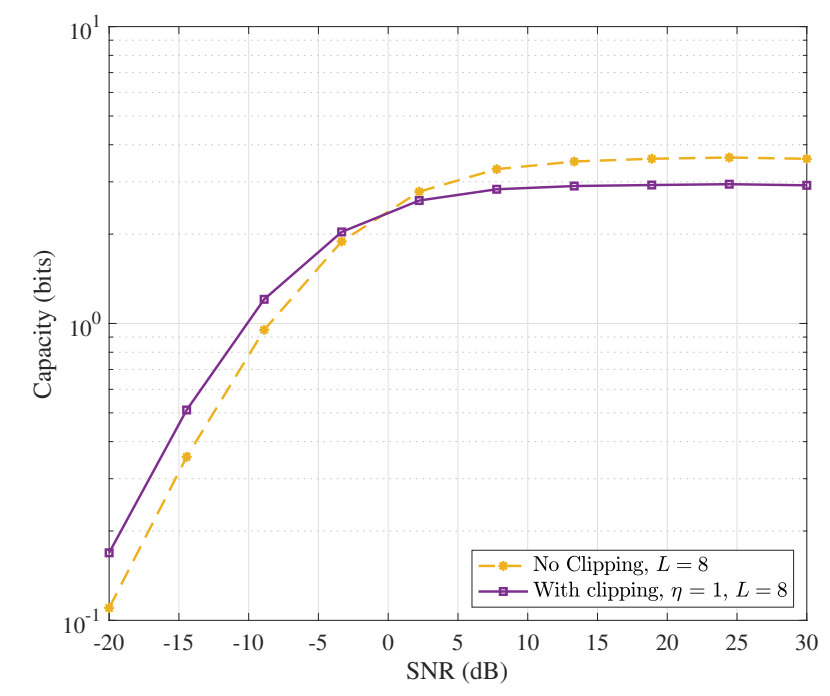

Fig. 4. Capacity results for a single-input single-output CP-DSSS channel.

\section{REFERENCES}

[1] A. Aminjavaheri, A. RezazadehReyhani, R. Khalona, H. Moradi, and B. Farhang-Boroujeny, "Underlay control signaling for ultra-reliable low-latency iot communications," in 2018 IEEE International Conference on Communications Workshops (ICC Workshops), 2018, pp. 1-6.

[2] H. Moradi and B. Farhang-Boroujeny, "Underlay scheduling request for ultra-reliable low-latency communications," in 2019 IEEE 2nd $5 G$ World Forum (5GWF), Dresden, Germany, Sept. 30 - Oct. 2, 2019, pp. 28-33.

[3] A. Stevens, H. Moradi, and B. Farhang-Boroujeny, "Ultra-reliable lowlatency control signaling in a factory environment," in 2020 IEEE $6 G$ Summit, Levi, Lapland, Finland, Mar. 17-20, pp. 1-6.

[4] S. Jenkins, B. A. Kenney, A. Majid, H. Moradi, and B. FarhangBoroujeny, "CP-DSSS: A novel waveform for multiple access in IoT," in 2020 IEEE 6 G Summit, Levi, Lapland, Finland, Mar. 17-20, pp. 1-6.

[5] B. A. Kenney, S. Jenkins, A. Majid, H. Moradi, and B. FarhangBoroujeny, "Cyclic prefix direct sequence spread spectrum capacity analysis," in 2020 IEEE Vehicular Technology Conference, accepted.

[6] B. A. Kenney, A. Majid, H. Moradi, and B. Farhang-Boroujeny, "Multiuser capacity for cyclic prefix direct sequence spread spectrum with linear detection and precoding," in 2020 IEEE Vehicular Technology Conference, accepted.

[7] H. Sari, G. Karam, and I. Jeanclaud, "Frequency-domain equalization of mobile radio and terrestrial broadcast channels," in 1994 IEEE GLOBECOM. Communications: The Global Bridge, 1994, pp. 1-5 vol.1.

[8] H. Sari, G. Karam, and I. Jeanclaude, "Transmission techniques for digital terrestrial tv broadcasting," IEEE Communications Magazine, vol. 33, no. 2, pp. 100-109, 1995.

[9] M. V. Clark, "Adaptive frequency-domain equalization and diversity combining for broadband wireless communications," IEEE Journal on Selected Areas in Communications, vol. 16, no. 8, pp. 1385-1395, 1998.

[10] N. Benvenuto and S. Tomasin, "On the comparison between ofdm and single carrier modulation with a dfe using a frequency-domain feedforward filter," IEEE Transactions on Communications, vol. 50, no. 6, pp. 947-955, 2002.

[11] N. Benvenuto, R. Dinis, D. Falconer, and S. Tomasin, "Single carrier modulation with nonlinear frequency domain equalization: An idea whose time has come-again," Proceedings of the IEEE, vol. 98, no. 1, pp. 69-96, 2010. 